\title{
Conference report "\#Communication \#(R)Evolution Changing Communication in a Digital Society": DACH 21 - Three-Country Conference on Communication Science (DGPuK, ÖGK, and SGKM)
}

\author{
Philipp Bachmann, Lucerne University of Applied Sciences and Arts, Institute of Communication \\ and Marketing IKM, Switzerland \\ philipp.bachmann.01@hslu.ch
}

In recent years, there has hardly been a conference in communication science without the buzzwords of digitization and transformation in its title. In an effort to stand out from the crowd, conference titles end up adorned with complementary buzzwords like "disruption," "datafication," "platformization," and "artificial intelligence."

These conferences frequently follow the same pattern: Papers and presentations are packed into individual panels by way of abstracts and keywords. Researchers then present their more or less elaborate empirical analyses to one other usually involving a great deal of statistics. Since these papers and presentations are based on a variety of theoretical foundations, methodologies, and statistical analyses, the level of mutual knowledge acquisition is often negligible. There are no common pillars - be they theoretical or empirical.

The Three-Country Conference hosted by Deutsche Gesellschaft für Publizistik- und Kommunikationswissenschaft (DGPuK), Österreichische Gesellschaft für Kommunikationswissenschaft (ÖGK), and Schweizerische Gesellschaft für Kommunikations- und Medienwissenschaft (SGKM) in Zurich from 7 to 9 April 2021 made an effort to counterbalance that trend.

The call for papers itself reflected on how society's digital transformation cannot - as is often attempted - be described by looking in from the outside. That is because communication science itself comprises part of society. To put this in other words: Since communication science is carried out in (digital) so- ciety, digital transformation also affects communication science itself. Against this background, the conference was entitled "\#Communication \#(R)Evolution Changing Communication in a Digital Society" and the topics addressed were defined in four key points: Theory (topic 1); methodology (topic 2); empirical research (topic 3 ); and self-reflection, i. e., the significance of self-comprehension and the role of our discipline (topic 4).

The insight that digital transformation also affects communication science itself was demonstrated not least by the fact that - due to the Corona pandemic the conference could not be held in $\mathrm{Zu}$ rich as had been planned, but rather took place via a virtual platform. In this context, the organizers Mark Eisenegger, Thomas N. Friemel, Mike S. Schäfer, and Thomas Zerback (Scientific Steering Committee) as well as Bettina Lennström and Olga Tartakovski (Conference Coordination) deserve a great deal of recognition for not simply doing a 1:1 transition of the originally planned analog conference into the digital realm. Instead, they leveraged the many opportunities that a digital conference has to offer.

One example to that end is how participants pre-recorded their presentations and uploaded them to the platform. Doing so made it possible for the presentations to be viewed in advance. Thus, all 31 panels were able to make better use of their time together. This worked extremely well in the panels which I personally attended. The platform was designed with great attention to detail. The organizers created brief videos to illustrate how to do a check-in or enter a conference room. In other words: 
Having experienced a little over one year of teaching online, the organizers drew the proper conclusions on how an online event should be implemented.

The organizers likewise also displayed dexterity with the selection of the keynote speaker. Dietram Scheufele of the University of Wisconsin-Madison and Morgridge Institute for Research got to the heart of the conference's theme in his keynote address. The title of the lecture was: "New instruments, paradigms, and information ecologies: the (ongoing) growing pains of our discipline." Scheufele, too, believes that communication science is facing a fundamental social transformation. In contrast to past advents such as radio or television, the Internet is not merely another new medium, but rather a new media environment which is hyper-selective and algorithm-driven. One consequence being: "Paper newspaper will die. Period. Algorithmic free press is dying out." $\mathrm{He}$ also cited how digital transformation is turning communication science itself into a "discipline in flux". Accordingly, "computational social sciences" are going to become more important across all disciplines. He noted how communication science still has room for improvement here as compared to other disciplines. $\mathrm{He}$ further postulated that open science is going to grow in relevance, as has also been illustrated in the "Agenda for Open Science in Communication” (Dienlin et al., 2021).
Somewhat neglected, however, was the question of how qualitative approaches align with Scheufele's vision of computational and open communication science.

In summary, the organizers did a fantastic job in establishing common ground far beyond theoretical and methodological issues, and well beyond the professional societies of DGPuK, ÖGK, and SGKM. In addition to Scheufele's keynote, the conference dinner will also be held in fond memory by the more than 500 participants from 16 countries. In advance, the organizers had sent out small packages to attendees featuring a fondue set with cheese, baby pickles, and Linzer tarts. Because despite all the digitalization and transformation going on out there - when it comes to eating and drinking, the analog and classic choices do continue to be the best. Thus, DACH 21 has succeeded in standing out from the crowd of online conferences without having to make any mention of "disruption," "datafication," "platformization," or "artificial intelligence."

\section{Reference}

Dienlin, T., Johannes, N., Bowman, N. D., Masur, P. K., Engesser, S., Kümpel, A. S.... de Vreese, C. (2021). Agenda for open science in communication. Journal of Communication, 71(1): 1-26. https://doi. org/10.1093/joc/jqz052 